\title{
Análisis teórico-reflexivo sobre los factores que intervienen en la calidad de los aprendizajes y práctica docente
}

Theoretical and reflective analysis of the factors affecting the quality of learning and teaching practice

Recibido 26 marzo 2015 • Aceptado 20 noviembre 2015 • Corregido 27 noviembre 2015

\author{
Guillermo Andrés Rodríguez Molina ${ }^{1}$ \\ Escuela Cerro Cornou \\ Talcahuano, Chile \\ guiandres.rm@gmail.com
}

\begin{abstract}
Resumen. El presente artículo es el principio del análisis teórico-reflexivo del estado del arte de investigaciones y literatura actual sobre la escuela, procesos pedagógicos, el profesorado y la calidad de los resultados académicos y de aprendizajes de los estudiantes. Se contextualizan los principales temas empíricos y teóricos de una investigación en curso que tiene como objetivos: a) analizar los procesos de gestión curricular y evaluación que influyen en la calidad de los aprendizajes y prácticas docentes en las organizaciones educativas, b) diseñar un modelo de gestión pedagógica dinámico y variable que contribuya al mejoramiento continuo de la calidad de los aprendizajes y prácticas docentes en las organizaciones educativas. Se trabaja con una metodología mixta, la cual permite converger o corroborar los datos recolectados, entregando los insumos técnicos para el diseño del modelo de gestión pedagógica, esperando dar respuesta a las siguientes interrogantes de investigación: ¿Cuál es la influencia en la calidad de los aprendizajes y las prácticas docentes de la gestión pedagógica: curricular y evaluativa en las organizaciones educativas? Y, ¿qué impacto tendría el desarrollo y aplicación de un modelo de gestión pedagógica en la calidad de los aprendizajes y prácticas docentes?
\end{abstract}

Palabras claves. Docente; calidad de la educación; gestión pedagógica; gestión curricular; procesos pedagógicos; reforma de la educación

Abstract. This article is the beginning of reflective-theoretical state of research and current literature on school, teaching processes, teachers and the quality of academic results and analysis of student learning. In which the main empirical and theoretical issues in an ongoing investigation aims are contextualized "analyze the processes of curriculum management and evaluation that influence the quality of learning and teaching practices in educational organizations" and "Designing a model

1 Doctorando en Educación, Universidad Autónoma de Barcelona - España. Magister en Psicología, Universidad de Concepción - Chile. Profesor de Educación General Básica, Jefe Unidad Técnica Escuela Cerro Cornou-Talcahuano, Chile. Se ha desempeñado como profesor de aula en distintos establecimientos educaciones, ha dictado clases de didáctica en la Universidad de Concepción y participado de coinvestigador en distintos proyectos de investigación. Además, tiene publicaciones como autor y co-autor en distintas revistas científicas. Actualmente se desempeña como jefe de la unidad técnica pedagógica y es doctorando en educación de la UAB, desarrollando su plan de investigación en gestión curricular y prácticas pedagógicas en centros educativos. 
Dynamic teaching and equity management that contributes to continuous improvement of the quality of learning and teaching practices in educational organizations "will work with a mixed methodology, which will converge or corroborate the data collected, providing technical inputs to the model design pedagogical management, hoping to answer the following research questions What is the influence on the quality of learning and teaching practices of educational management: curricular and evaluative in educational organizations? And what impact would the development and application of a model of educational management in the quality of learning and teaching practices?

Keywords. Teaching; quality of education; educational management; curriculum management; pedagogical processes; Education Reform

\section{Introducción}

El desafío actual de los sistemas educativos, en todas sus etapas, es enorme; esto son las transformaciones, tanto de forma como de fondo, que se llevan a cabo hoy en día y están movilizando a todo el espectro de la sociedad, especialmente a los actores educativos. Sin embargo, no solo en Chile está presente el desafío de dar un nuevo giro a los sistemas educativos, pues la Organización de las Naciones Unidas para la Educación, la Ciencia y la Cultura (UNESCO, 2014) ha declarado que los sistemas de educación vigentes no son totalmente sostenibles en relación con el desarrollo humano actual. De ahí, que es necesario establecer un marco de referencia para la educación, basado en esta como un derecho humano, social, un bien en sí mismo, eliminando toda forma de segregación y discriminación. Este marco de referencia debe ser capaz de responder a las prioridades de toda la sociedad, en su conjunto.

A partir de esto, es donde las organizaciones educativas: escuelas, liceos y universidades, deben concentrar y alinear sus estrategias para dar respuesta satisfactoria a la necesidad de una nueva educación.

Por ello, es necesario primero reflexionar acerca de los nudos críticos que se están sucediendo en nuestro sistema educacional; luego dar una profundidad teórica de aquellas variables específicas que influyen en calidad de los aprendizajes de nuestros estudiantes; seguido de la gestión pedagógica, desde lo curricular hasta lo evaluativo, que llevan a cabo los profesores y profesoras, de acuerdo con la evidencia evaluativa vigente; y por último, en la etapa terminal de la investigación, entregar una propuesta concreta con fundamentos serios sobre aquellos aspectos que deberían ser mejorados para lograr los cambios necesarios a nivel de escuela.

El presente artículo teórico-reflexivo, se enmarca dentro del análisis de diversas investigaciones, evidencias y literatura actual sobre el estado de la educación chilena y otros sistemas educativos, específicamente con aquellos procesos de gestión pedagógica (curricular y evaluativa) que influyen tanto en la calidad de los aprendizajes de los estudiantes como en las prácticas docentes de las heterogéneas organizaciones educativas y en un marco general de la investigación que se lleva a cabo. 


\section{Segregación escolar y social en Chile}

La evidencia nacional reciente señala que, en Chile, existe segregación socioeconómica en el sistema escolar, lo cual se presenta como un desafío que debe serenfrentado por la política pública. Las diversas investigaciones han mostrado que el sistema escolar presenta una segregación que fluctúa entre moderada y alta (Ministerio de Educación [MINEDUC], 2012). Sin embargo, dicha evolución cambia en negativo, dado que la segregación en el sistema escolar chileno ha experimentado un aumento a lo largo de la última década (Valenzuela, Bellei y De Los Ríos, 2011).

A pesar de los datos disímiles, la conclusión evidente sigue siendo que el sistema educacional chileno, tal cual como se estructura, es un agente segregador a pesar de los catalizadores de política pública que se han implementado; no hay un avance significativo al respecto, considerando la evidencia y el informe de la Oficina para la Cooperación y Desarrollo Económico (OCDE), del año 2004.

La segregación escolar es un freno social para las aspiraciones de crecer con calidad y equidad. El informe de resultados del Estudio Internacional de Progreso en Comprensión Lectora y Estudio Internacional de Tendencias en Matemáticas y Ciencias (PIRLS-TIMSS, 2011)2 para Chile, destacó que la diferencia en la tendencia media entre establecimientos municipalizados y particulares pagados, había caído en la prueba de Matemática, aunque no en Ciencias. Una conclusión parcial de ello, es que existe un avance en la equidad intergrupal. Sin embargo, no hay evidencia alguna con respecto a qué fuerzas están actuando, en este caso, de la disminución de brechas en puntajes: ¿profesores, estudiantes, padres, política pública, etc.? El estudio en cuestión, no determina ninguna variable, y la evidencia ministerial y académica, aún no tiene resultados al respecto.

En este ámbito, se ha hecho una simulación estadística, en el año 2013, del desempeño de los estudiantes, implementando programas focalizados; estos resultados concluyen que la "política no focalizada podría ser más eficiente en el elevar el desempeño promedio de todo el sistema a corto plazo" (MINEDUC, 2013a, p. 5). Estos programas focalizados, Plan de Apoyo Compartido (no implementado oficialmente desde 2014), Plan de Formación de Directores y Beca Formación de Profesor, sin lugar a dudas, no entregan un auspicioso futuro en cuanto al desempeño de los estudiantes en todas las áreas de aprendizaje y aumentan la segregación entre estudiantes.

\section{Organizaciones educativas como origen del cambio}

Posiblemente uno de los peores pecados de la educación, y en especial de las organizaciones educativas, es la autocomplacencia, creer que se ha hecho todo bien y no necesita mejoras. Si los cambios de toda índole (sociales, demográficos, económicos, tecnológicos, etc.) son constantes,

2 Ver Resultados PIRLS-TIMSS 2011, Chile, en: http://www.mecd.gob.es/dctm/inee/internacional/pirlstimss2011vol1-1. pdf?documentld=0901e72b81825be4 
los educativos también deben serlo. Impulsar y sostener estos procesos de cambio en las escuelas requiere entusiasmo, voluntad, compromiso y ganas de aprender a hacer mejor las cosas (Murillo y Krichesky, 2012), estableciendo primero que todo, un amplio proceso democrático para desarrollar un plan estratégico, en el cual el sistema identifique las prioridades claves.

Con base en estos cambios, orientados a la mejora continua, está el considerar la escuela u organización escolar como parte de un sistema educativo integrado, el cual posee una dinámica propia; una dinámica y unas fuerzas que se imponen a quienes en ellos se integran, a quienes intentan introducir modificaciones en estos y a quienes con ellos se relacionan. Los sistemas educativos, integrados por grupos de personas con sus intereses y puntos de vista propios, no parten de esta dinámica.

Por lo anterior, es que la organización educativa es una cultura escolar con sentido propio, constituida por un conjunto de teorías, ideas, principios, normas pautas, rituales, inercias, hábitos y prácticas sedimentadas a lo largo del tiempo en forma de tradiciones, regularidades y reglas de juego no puestas en entredicho, y compartidas por sus actores en el seno de las instituciones educativas. Estas prácticas proporcionan estrategias para integrarse e interactuar con diversas organizaciones, llevar a cabo el trabajo de aula y adaptarse a las múltiples reformas, de acuerdo con su cultura y contexto.

Para ello, es necesario mirar hacia una escuela abierta, flexible, centrada en la institución, es decir, una escuela autónoma que pueda establecer "sus propios planteamientos en razón de su propio contexto, de su historia institucional y su cultura organizativa. Su identidad queda así ligada a su capacidad y sensibilidad para analizar la realidad y para dar respuesta a demandas e inquietudes existentes" (Gairín, 2008, p. 83).

Para lograrlo, la institución debe considerar el factor sociocultural, el cual ejerce presión para generar nuevos procesos educativos autónomos y de cambio. A pesar de ello, la escuela no debe adaptarse al entorno; debe estudiarlo a fondo y generar los procesos de transformación. La escuela no debe ser a imagen y semejanza del medio en el que se encuentra, pues se quedaría estática; por el contrario, desde un diagnóstico real del contexto sociocultural, debe definir unos fines que le permitan, con axialidad, encontrar respuestas nuevas a las condiciones nuevas del continuo devenir. Si los centros educativos no se contextualizan en sus entornos culturales, reales e ideales no producirán transformaciones, retardarán los cambios educacionales y darán continuidad a modelos tradicionales y a paradigmas ya superados en otras áreas del conocimiento.

Por tanto, las escuelas con una cultura propia que se orienta a los procesos de mejora en todos sus ámbitos (aprendizaje, profesores y gestión pedagógica) implica un desarrollo de una forma de pensar y actuar que promueva la mejora constante de los procesos que se dan a largo plazo y son propios de la organización, las cuales requieren al menos conocer la naturaleza del cambio, así como las fases por las que atraviesa la escuela, convirtiendo espacios que fomenten no solo el desarrollo integral de los estudiantes sino también, y como medio para ello, el desarrollo profesional de los profesores y profesoras. 
Así, las organizaciones que aprenden son las que están mejor preparadas para afrontar los procesos de cambio y mejora, indispensables para hacer de toda organización una institución de calidad (Senge, 1990).

\section{¿Cuál es el rol del cuerpo docente?}

La formación de profesoras y profesores en la práctica pedagógica es clave a la hora de determinar el desempeño y calidad de los aprendizajes de todos los estudiantes, ya que son los catalizadores profesionales para realizar los puentes didácticos y curriculares, desarrollando en los niños y niñas las habilidades, competencias y actitudes necesarias para enfrentar la llamada sociedad dinámica del conocimiento. Así lo revela la investigación realizada por Coleman, Campbell, Hobson, McPartland, Mood, Weinfeld y York (1996), quienes manifiestan el importante rol que juegan los docentes y la magnitud de la influencia en los resultados de los estudiantes. Goe (2007), después de varias revisiones empíricas, concluye que la calidad docente, medida de diversas formas, afecta positivamente en los estudiantes. El informe Mckinsey (2007) manifiesta la importancia de los docentes estableciendo que la calidad de los sistemas educativos no puede exceder la calidad de su profesorado. Complementando las investigaciones ya mencionadas, el estudio Teaching and Learning International Survey (TALIS) de la OCDE (2013), señala que el cuerpo docente es un elemento clave en la educación, la calidad de este es uno de los factores que más influye en el aprendizaje y rendimiento del estudiantado. Por lo tanto, apoyar y fortalecer la profesión docente de calidad debe ser una prioridad de cualquier sociedad y para conseguir este objetivo, es fundamental conocer el ambiente de aprendizaje de las organizaciones educativas y las condiciones de trabajo del profesorado.

En este aspecto, en Chile, desde el año 2003, comenzó la implementación del Sistema de Evaluación Docente ${ }^{3}$ cuyo objetivo es fortalecer la profesión docente, favoreciendo el reconocimiento de las fortalezas y la superación de las debilidades de los docentes, con el fin de lograr mejores aprendizajes de sus alumnos y alumnas; este sistema, voluntario en su inicio y obligatorio en la actualidad, solo se aplica al profesorado del sistema municipal de educación, dejando por fuera a gran parte del resto de docentes, remunerados con la subvención estatal.

Los instrumentos empleados en dicha evaluación son variados: portafolio, pauta de autoevaluación, entrevista de evaluador para el informe de referencia de terceros. Estos son construidos con base en los dominios, criterios y descriptores que provee el Marco para la Buena Enseñanza (actualmente en revisión e inclusión de estándares de desempeño). Durante el año 2011, se realizó una revisión del sistema por parte de expertos de la OCDE, los cuales señalan que se debe mejorar el sistema en tres puntos: mejorar los mecanismos de información al cuerpo docente relativos a las indicadores de desempeño y revisión de los indicadores, de

3 Reglamento sobre Evaluación Docente, Decreto №192 de Educación del 30 de agosto de 2004, en http://www.cpeip.cl/usuarios/cpeip/ File/2013\%20documentospsp/reglamentoevaluaciondocente.pdf 
modo que reflejen la última evidencia respecto a las buenas prácticas docentes; fortalecer la función formativa del proceso de evaluación, potenciando las formas de retroalimentación; y revisar los instrumentos de evaluación y procedimientos utilizados.

En este contexto, es necesario preguntarse: ¿Cómo se relaciona el desempeño de cada profesor o profesora, de acuerdo con las dimensiones e instrumentos del sistema de evaluación, con el rendimiento y aprendizajes de los estudiantes? Hay planteamientos de Goe y Stickler (2008) y Rivkin, Hanushek y Kain (2005), quienes señalan que no hay evidencia contundente sobre qué aspectos de la calidad docente son los más importantes para predecir el aprendizaje de los estudiantes.

Entonces, es válido profundizar sobre aquellas dimensiones de la evaluación docente que podrían influir en el rendimiento y aprendizaje. Para ello, se analizó la información del texto La evaluación docente en Chile (Manzi, González y Zun, 2011) que evidencia los resultados del sistema de los años 2007 - 2010. Ahí, se señala que las dimensiones que tienen un promedio más bajo son: calidad de la evaluación de la unidad; reflexión a partir de los resultados y reflexión pedagógica. Si se reaiza un análisis por ámbitos evaluados, los resultados más deficientes son: organización de las situaciones de aprendizaje, interacción pedagógica, evaluación del aprendizaje y reflexión pedagógica.

Observando las expectativas relativas a la tarea de educar y al rol del docente, todo ha cambiado considerablemente. Para mencionar algunos de los aspectos más destacados en las políticas educativas y los currículos contemporáneos, hoy se aboga por una enseñanza que favorezca el aprendizaje activo, centrada en las necesidades y expectativas de los estudiantes considerados como los protagonistas principales en el proceso de construcción y regulación de sus aprendizajes, que tome en cuenta que en todo proceso de aprendizaje las dimensiones cognitiva, ética y emocional están interrelacionadas y no pueden ser disociadas arbitrariamente; que se adapte a la diversidad de características y estilos de aprendizaje de los estudiantes; que facilite la comprensión y la aplicación de conocimientos más que su acumulación; y que haga el mejor uso posible del potencial de las tecnologías de la información y comunicación.

Las modalidades de formación y de desarrollo profesional de los docentes, así como su rol tradicional, se encuentran frecuentemente en el centro de las críticas y se nota una desconfianza creciente hacia su profesionalismo.

Al mismo tiempo, se reconoce que el docente sigue teniendo un papel clave como facilitador de las experiencias y de los procesos de aprendizaje, y que su tarea se ha vuelto más compleja. Sin embargo, sobrecargados por una pluralidad de funciones y responsabilidades que van más allá de lo pedagógico, llamados a resolver problemas sociales de toda índole, encerrados en su aula, sin posibilidad de aprender de otros y a su vez compartir su experiencia, limita las posibilidades de construir un continuum entre las prácticas individuales de aula y los aprendizajes que debieran acompañar esas prácticas, abrumados por listados interminables de estándares de desempeño y objetivos a alcanzar, presionados por rendir cuentas sobre resultados que no se contextualizan, 
obligados a aceptar condiciones de trabajo frecuentemente precarias e insatisfactorias; los docentes tienen que cohabitar con las inconsistencias y tensiones que atraviesan las propuestas curriculares (en cuyo diseño muchas veces no participan) y pueden, fácilmente, llegar a perder de vista el aspecto más desafiante y apasionante de su labor: cómo educar a los ciudadanos del mañana.

En este sentido, donde los cambios en la política educativa se vislumbran con mayor nitidez, es cuando es necesario considerar el desarrollo de una política de formación continua, centrada en la didáctica de la disciplina y el acompañamiento en la implementación de mejoras en las prácticas pedagógicas en las salas de clase, las cuales juegan un papel significativo en el grado de aprendizaje de los estudiantes.

Existe evidencia en la literatura acerca de la efectividad de las prácticas activas de enseñanza, así como de la necesidad de su correcta utilización para que generen un aprendizaje realmente efectivo. Los factores que determinan el uso, por parte de los profesores, de las prácticas de enseñanza activa son muy variados. No obstante, si un profesor tiene sólidos conocimientos pedagógicos de las materias que enseña, puede estar más preparado para utilizar prácticas activas de enseñanza.

No cabe duda que el rol docente y principalmente, su práctica pedagógica, son un pilar por antonomasia fundamental para el logro de los objetivos de todo sistema educacional, el rendimiento escolar, de los aprendizajes y la calidad educativa. Las responsabilidades que tiene, no solo en Chile sino en muchos países, puede decirse que los profesores están más que nunca en el punto de mira. Este aumento de responsabilidades proporciona una buena oportunidad para que la sociedad, en general, comprenda las condiciones en las que sus profesores trabajan y las barreras o impedimentos que encuentran para enseñar a la siguiente generación de ciudadanos activos.

\section{La calidad educativa, desafío y oportunidad}

La preocupación de nuestro tiempo ha dejado de ser, únicamente, el acceso generalizado a la enseñanza básica y media, para centrarse en que ese acceso se produzca con calidad y se extienda a lo largo de toda la vida, haciendo posible que las personas aspiren a mejores condiciones de vida y que las sociedades se desarrollen equitativa y sosteniblemente.

Hoy en día, se extiende el convencimiento de que la calidad es parte inherente del derecho a la educación, siendo una de las condiciones esenciales, como una cualidad constitutiva de ella.

La evidencia, tanto en Chile como en el extranjero, muestra el poco avance que han tenido los resultados de las evaluaciones estandarizadas (OCDE, 2013; MINEDUC, 2013a; MINEDUC, 2012), a pesar de las reformas impulsadas y los cuantiosos recursos que se han invertido; sumado a ello, los resultados dispersos que se aprecian a nivel de evaluación docente y práctica efectiva en las aulas, están provocando que los estudiantes no logren aprender lo que necesitan y con la calidad que se merecen. Entonces, ¿bajo qué aspectos se puede decir que la educación es de calidad, o más bien, cómo podemos entregar una educación de calidad para todas las personas? 
La calidad educativa es un desafío de los tiempos actuales y es clave para que la educación promueva el desarrollo. Sin embargo, esta calidad no será posible si no existen acciones que se focalicen en aspectos relevantes como: procesos de enseñanza-aprendizaje (el currículum, la metodología, didácticas apropiadas, recursos de apoyo y rol docente); los procesos de gestión (proyecto de la organización educativa, papel del director y equipo directivo, infraestructura) y los procesos de convivencia y construcción de ciudadanía (estado de la vida saludable de los estudiantes, autoimagen de los estudiantes, expectativas y las relaciones escuela-comunidad).

Un aspecto central para lograr la calidad, son los procesos que se llevan a cabo en la gestión curricular. Esta vinculación de la gestión con las áreas propias del quehacer educativo, están en sintonía con la gestión pedagógica, pero se deben distinguir aquellas que afectan el proceso sistémico y complejo de la gestión propiamente como tal. Por ello, cuando se hace la vinculación gestión y currículo, se focaliza en la gestión de los saberes a enseñar en las escuelas y la construcción de experiencias formativa para que los estudiantes se apropien de estos saberes previamente seleccionados (Falconi, 2008).

Bajo una dimensión aún más concreta, la gestión curricular describe las políticas, procedimientos y prácticas que lleva a cabo el director, el equipo técnico-pedagógico y los docentes de la organización educativa para coordinar, planificar, monitorear y evaluar el proceso de enseñanza-aprendizaje (MINEDUC, 2013b).

Esta organización de los saberes dentro de la gestión pedagógica y en torno al currículo, es pensar un texto que oriente prácticas pedagógicas vinculadas entre ellas con sentido y coherencia, construidos, en forma colectiva, entre el líder pedagógico y el cuerpo pedagógico.

La gestión curricular se comprende como parte del marco de la gestión educativa, implica construir saberes teóricos y prácticos en relación con la organización del establecimiento escolar, aspectos administrativos, actores que forman parte de la institución y por supuesto, con el currículo escolar. En consecuencia, focaliza algunos de los aspectos incluidos en la gestión educativa en vistas a profundizarlos, ampliarlos, complejizarlos, completarlos; pero sobre todo, trata de abordar centralmente, los saberes vinculados en forma directa con la dimensión pedagógico-didáctica. Ello involucra volver a situar la escuela en torno a la enseñanza y al aprendizaje, lo que supone enfatizar en la gestión de los aprendizajes que son responsabilidad de toda institución educativa, como institución social. Asimismo, la gestión del currículum se vincula directamente con los procesos de toma de decisiones en relación con qué, cómo y cuándo enseñar y evaluar, pues constituyen actividades centrales que se desarrollan en el establecimiento escolar y que son diseñadas e implementadas por los docentes.

Dentro de los procesos referidos a la gestión curricular y que son llevados a cabo por el equipo de los unidades técnicas y/o liderazgo pedagógico, se encuentran, por una parte, la coordinación de los tiempos para la implementación curricular, que corresponde a la generación de las condiciones que permiten emplear, de manera efectiva, las horas de enseñanza 
disponibles para el logro de los objetivos académicos y formativos (Leithwood, Day, Sammons, Harry \& Hopkins, 2006; Robinson, Hohepa \& Lloyd, 2009; Weinstein \& Muñoz, 2012). Esto implica asignar estos tiempos de enseñanza mediante políticas de procedimientos (Murphy, 1990) que sirvan para organizar a los profesores y profesoras en su quehacer pedagógico, focalizando el trabajo y resguardando desviar el foco de las metas de aprendizaje, pudiendo concretarse así las actividades de enseñanza planificadas.

Por otra parte, la conducción curricular requiere que en la organización escolar se cuente con directrices para el trabajo pedagógico de los profesionales de la educación, lo que implica acordar y coordinar con ellos criterios y estándares en aspectos como la planificación, la evaluación o la política de asignación de tareas (Weinstein, 2009). Esto permite que los profesores dispongan una visión clara sobre cómo se debiese implementar la enseñanza (Marzano, 2012) y sigan lineamientos pedagógicos comunes para hacerlo, de manera coherente, entre niveles y áreas de aprendizaje.

Lo anterior, también incluye asesorar a los profesores sobre el uso de mejores prácticas y metodologías de enseñanza (Hallinger \& Murphy, 1985; Weber, 1996; Robinson et al., 2009; Weinstein \& Muñoz, 2012), asegurando la provisión de recursos pedagógicos y didácticos para la implementación curricular y evaluación, lo que implica, además de la facilitación de materiales y/o recursos de aprendizaje, que constituyan efectivamente un apoyo para el logro de los objetivos educativos.

Otro de los elementos referidos a la gestión curricular y liderazgo pedagógico mencionado transversalmente en la literatura, es la planificación curricular y de la enseñanza (Weber, 1996; Robinson et al., 2009; Bambrick-Santoyo, 2012; Weinstein \& Muñoz, 2012), proceso que constituye una herramienta fundamental para programar e implementar el trabajo pedagógico en el aula. En general, el proceso de planificación implica identificar los contenidos que resultan esenciales que todos los estudiantes aprendan, estimar el tiempo requerido para asegurar que, efectivamente puedan ser enseñados y secuenciarlos, y organizarlos para optimizar la experiencia de aprendizaje (Marzano, 2003).

El monitoreo del trabajo pedagógico o apoyo a los profesores mediante la observación de clases, es un proceso fundamental de la gestión curricular, ya que está directamente relacionado con el desempeño profesional docente y su mejora. Para ello, debiese hacerse en relación con prácticas concretas, proporcionar sugerencias y comentarios específicos sobre el proceso de enseñanza y aprendizaje (Murphy, 1990), incluyendo una revisión conjunta de las prácticas pedagógicas (Robinson et al., 2009) e identificar áreas de crecimiento profesional, con el fin de considerar pasos específicos para la mejora. Además, es preciso asegurar el desarrollo del docente y de las metas de crecimiento establecidas. Esto bajo un espacio de retroalimentación con carácter reflexivo (Marzano, 2003), donde tanto el profesor como el líder pedagógico revisen activamente las prácticas pedagógicas.

Un elemento complementario al proceso de gestión curricular anterior, tiene relación con la creación de espacios para la discusión técnica, promoviendo el debate e intercambio 
profesional, focalizados en la reflexión de las prácticas pedagógicas implementadas, analizadas de manera crítica y constructiva, intercambiar ideas, estrategias, recursos, soluciones, buscar y entregar apoyo a los demás profesionales, dando lugar a un trabajo colegiado (Marzano, 2003; Robinson et al., 2009; Marzano, Pickering \& Heflebower, 2011) donde prime el bien colectivo y trato respetuoso, donde se aprenda en conjunto, se valore el bien colectivo, se aprenda en equipo, se compartan fracasos y errores (Elmore, 2000), de manera que favorezca una cultura de co-aprendizaje y construcción del conocimiento colectivo.

Por último, el proceso de monitoreo de la implementación del currículo y el aprendizaje, es la finalización y punto de partida de un sistema de proceso curricular llevado a cabo por el liderazgo pedagógico y/o unidades técnicas. Este proceso de gestión curricular se ocupa de supervisar la cobertura curricular mediante visitas al aula, verificando planificaciones de clases y reuniones en conjunto con los docentes (Marzano, 2003). Adicional a ello, la observación de los resultados de aprendizaje, análisis de evaluaciones estandarizadas e interpretaciones de los datos entregados.

Examinar la implementación del currículo permite verificar su ejecución adecuada para que los estudiantes alcancen los objetivos de aprendizaje, y tomar las decisiones curriculares y pedagógicas junto al cuerpo docente. Elmore (2010) sostiene que un monitoreo y análisis adecuado de los procesos de enseñanza-aprendizaje requiere identificar relaciones causaefecto entre lo que se espera lograr y lo que realmente ocurre. Para ello, debiese analizarse, permanentemente, la forma en que las prácticas pedagógicas se traducen en aprendizaje en el contexto escolar particular, para lo cual propone el método de juntas pedagógicas.

En 2008, los ministros de educación de la región latinoamericana impulsaron el proyecto Metas Educativas 2021, con el que se pretendía mejorar la calidad de la educación y el currículum escolar, considerando la importancia de la calidad educativa, vista desde tres puntos de vista: desarrollo económico, cohesión social y eficiencia escolar (OEI, 2008).

Pero, ¿qué se entiende por calidad? Este concepto está íntimamente relacionado con la percepción que se tiene del mundo y los valores que se defienden, pudiendo significar cosas diferentes para distintos observadores y grupos de interés. La educación siempre estará sometida a debates ideológicos, pues en ella se concentran las expectativas, valores y visiones en la relación con la sociedad en la cual vivimos. Es, sobre todo, el trasfondo ideológico que encierra lo que hace que la calidad educativa no sea un concepto neutro y que no resulte sencillo definirlo.

La UNESCO (2004), en su informe Enseñanza para Todos, realizó una clasificación de los diversos enfoques que aproximarían a entender las polisémicas definiciones de calidad educativa que pueden encontrarse. Las corrientes son:

- Enfoques conductistas

- Enfoques humanistas 
- Enfoques críticos

- Enfoques autóctonos

A partir de la diversidad de enfoque, se multiplican las definiciones de calidad y se hace complejo y multidimensional una única definición, la cual debe abarcar y vincular la educación como formación integral de la persona, el rendimiento académico, los factores asociados a la formación de los estudiantes, la justicia educativa, cuantificación y cualificación educativa y la diferenciación de formulaciones teóricas y prácticas. Básicamente es un ejercicio que debe llevar a explicitar qué tipo de escuela queremos, sobre qué valores la construiremos, qué procesos pedagógicos se privilegiarán y qué vínculos se establecerán entre calidad educativa y proyecto de sociedad.

La OREALC/UNESCO (2007) sustenta una definición considerando distintas variables y la define como:

La calidad de la educación en tanto derecho fundamental, además de ser eficaz y eficiente, debe respetar los derechos de todas las personas, ser relevante, pertinente y equitativa. Ejercer el derecho a la educación es esencial para desarrollar la personalidad e implementar los otros derechos. (p. 7)

En una primera síntesis con respecto a la calidad educativa, se resalta la multidimensionalidad, la cual abarca tres focos relacionados entre sí, desde el cual se configura un cuadro perfecto del desarrollo educativo integral: educación de calidad, escuelas de calidad y sistemas educativos de calidad.

En cuanto a la educación de calidad, el conocido informe de Delors, Mufti, Amagi, Carneiro, Chung, Geremek, Gorham, Kornhauser, Manley, Padrón-Quero, Savané, Singh, Stavenhagen, WonSuhr y Nanzhao (2000), La Educación encierra un tesoro, define cuatro pilares de la educación (se entiende, de calidad) para este siglo:aprender a conocer, aprender a ser, aprender a hacery aprender a vivir con otros. A pesar del consenso encontrado por muchos expertos y países en cuanto a los estándares mínimos para definir la calidad educativa, existe una corriente latinoamericana que incorpora una visión ausente en el informe de la UNESCO (2014), que apunta a los valores como el sentido comunitario de vida, la multiculturalidad e interculturalidad o la apertura y valoración de formas de conocimiento, que son propias de las culturas latinoamericanas y no se han posicionado de manera significativa en el debate sobre la calidad.

Las organizaciones educativas de calidad, también llamadas escuelas efectivas a principios del 2000, bajo un prisma de resultados estandarizados y un modelo de competencia, deben ser aquellas que proporcionan diferentes opciones, caminos y modalidades, equivalentes en calidad, para atender a la diversidad de necesidades de las personas y de los contextos en los que tienen lugar los procesos de enseñanza y aprendizaje. Deben apuntar a la sistematicidad y perdurabilidad de los aprendizajes, diferenciación de sus prácticas y proyectos de aula, donde la participación de 
la comunidad escolar en su conjunto, entiéndase estudiantes, padres, apoderados, profesores, asistentes y líderes, busquen la satisfacción de todos los agentes educativos.

Por su parte, un sistema educativo de calidad, donde todos los aspectos de financiamiento y lineamientos de política pública deben orientar el trabajo de las organizaciones educativas y sus agentes. Un aspecto fundamental de un sistema de calidad tiene que ver con el tipo de persona que se quiere formar de acuerdo con las necesidades propias de un Estado y que se plasman en el currículum educativo, cuyo proceso de construcción ciudadana refleje el tipo de sociedad que se aspira forjar, comprometiendo la diversidad de instituciones y actores.

Es evidente que el currículum escolar no puede abarcar todo lo que es preciso aprender en todos los planos de la persona, por tanto, no hay algo sustancialmente nuevo en este proceso. Sin embargo, los cambios que están afectando a nuestras sociedades, las tensiones y dilemas que se han ido acumulando en busca de acuerdos sociales y políticos sobre el cómo, por qué y para qué educar, responden a las necesidades propias de las personas y sectores involucrados, han dado celeridad a los cambios. Actualmente, diversas evaluaciones que se han hecho a los currículos escolares de diferentes países, muestran que estos tienden a mirar más hacia el pasado que al futuro, basado en concepciones muy tradicionales del aprendizaje y de la enseñanza, privilegiando la acumulación de hechos, informaciones y saberes en lugar de favorecer la comprensión de lo que se puede hacer con esos saberes, dejando de atender los resultados del proceso educativo, expresados en términos de competencias genéricas o transferibles que los estudiantes deberían haber aprendido a desarrollar y aplicar al completar su educación.

Cerrar la distancia que frecuentemente separa las intenciones y las aspiraciones consignadas en los documentos curriculares de la realidad cotidiana de las escuelas y de lo que los estudiantes que efectivamente aprenden, es posiblemente uno de los desafíos principales de todos los sistemas educativos. Los resultados de las pruebas estandarizadas son útiles para identificar las brechas existentes y establecer algunas prioridades de política pública y reformas curriculares, pero solo tienen un valor en sí misma, ya que se focalizan en los resultados más que en los procesos y se concentran en algunas áreas de aprendizaje.

No cabe duda que es oportuno evaluar y medir el alcance del aprendizaje escolar en relación con criterios como estándares, objetivos y competencias, pero existe también mucha preocupación con respecto a un discurso dominante que valora la calidad y riqueza del aprendizaje, únicamente sobre la base de lo que se puede medir en un número muy limitado de disciplinas y con instrumentos estandarizados a una diversidad de estudiantes y organizaciones educativas, por muy fundamental que se considere (Amadio, Oportti y Tedesco, 2014). De hecho, existe el riesgo que las decisiones tomadas sobre una base tan restringida de evidencias distorsionan el sentido de la calidad educativa, cambios curriculares y las practicas docentes, lo cual visibilizaría la movilización del conocimiento y las particularidades del colectivo de estudiantes, el aula, la escuela y el sistema. 
En definitiva, si se quiere dar un giro a la efectividad curricular y alcanzar los objetivos propuestos, es necesario repensarlaestructura disciplinartradicional del currículum, laorganización de las experiencias de aprendizaje, las maneras de enseñar y los sistemas de evaluación.

Como la definición de calidad educativa es compleja en sí misma, hay algunas certezas a modo de conclusión que se van abriendo paso sobre lo que conviene a la calidad educativa, las cuales se agruparán en torno a tres ejes (Aguerrondo, 1993): Políticas y estrategias en el plano epistemológico (claridad de objetivos para la calidad y pertinencia en los currículos); Políticas y estrategias en plano pedagógico (el estudiante en el centro del proceso, la calidad ligada a la equidad, la calidad educativa comienza pronto, los docentes piedra angular, política lingüística adecuada) y Políticas apropiadas a lo organizativo-administrativo (la escuela en primer plano, recursos materiales que ayuden, evaluar para aprender y la calidad para todos). Esta agrupación, en cuanto insumo para desarrollar una definición más precisa, integral y completa de calidad educativa, lleva a un debate social y académico necesario. El concepto de calidad, va más allá de los restringidos límites de las evaluaciones estandarizadas, pues son la equidad, pertinencia educativa, integración social y participación lo que se encuentra sobre estos resultados. No es necesario emular a países con grandes niveles de igualdad, pues territorios como el nuestro, con grandes niveles de segmentación y desigualdad, deben refundar los principios tan básicos como los reflexionados.

Eneste sentido, cobra especial relevancia no perder devista los principios (equidad, integración, participación y calidad) que deben orientar y estar a la base de los procesos de mejoramiento de la calidad. Estos tienen que ver con equidad, calidad para todos y el fortalecimiento de la sociedad democrática que provea a los estudiantes de herramientas para su integración social y económica, permitiendo un ejercicio pleno de los derechos ciudadanos, no solo para adaptarse a la sociedad, sino también para tener herramientas para construir una más justa y libre.

\section{Conclusiones}

Las reflexiones empírico-teóricas del presente trabajo conducen por las variadas aristas que componen la educación. Sin embargo, el análisis se enmarcó dentro de los temas que se van a profundizar en la investigación, lo cual permitió situar las temáticas teóricas y empíricas más relevantes de los últimos años, tanto a nivel nacional como internacional, para triangular la información y allanar el camino del investigador en esta etapa de revisión teórica.

Nos encontramos con aspectos en los cuales el desarrollo de los sistemas educativos, especialmente el chileno, ha tenido poco avance a través de los años, la segregación escolar sin que todavía no haya un método único para poder medirla cuantitativamente, los estudios tanto nacionales como internacionales, desde el 2008 al 2011, demuestran que la evolución ha sido estadísticamente no significativa. 
Por otra parte, la escuela, los profesores y la calidad educativa son factores relevantes para el desarrollo óptimo, tanto de los aprendizajes como del rendimiento educativo de los niños y las niñas. La organización educativa, como centro donde confluyen estudiantes y familias con sus necesidades, puede propiciar el profesorado con sus prácticas pedagógicas que se podrían integrar y llegar a un modelo de gestión pedagógica que contribuya al desarrollo de prácticas efectivas; la escuela como mediadora social, conforman una cultura única y diversa que debe educarse y aprender de acuerdo con los múltiples desafíos internos y externos para responder, primero que todo, a los objetivos de su proyecto, luego a las necesidades pedagógicas de los estudiantes y entregar un desarrollo profesional docente en todas sus dimensiones a los profesionales de la educación. Implementando lo anterior la organización educativa volverá a ser el centro de integración social, creativo y autónomo que permitirá el desarrollo de los niños, niñas y jóvenes en sus múltiples dimensiones y que puedan contribuir a sus países desde sus intereses y oportunidades.

Finalmente, como último aspecto, se analiza en profundidad los alcances de la calidad educativa, los factores que determinan su ontología y definición hasta llegar a una comprensión del término y su aplicabilidad en las organizaciones educativas, buscando potenciar el desarrollo integral de las personas en todas sus dimensiones, valorando su individualidad y pertenencia sociocultural favoreciendo la apropiación y construcción personal y colectiva de conocimientos, actitudes y habilidades.

En definitiva, esta reflexión teórica-empírica lleva la tarea de cambiar las miradas actuales, tanto de las investigaciones como los diseños e implementación de políticas en sistema educativo, para que se puedan mejorar sustantivamente y alcanzar la promoción social entendida como proceso concientizador, transformador, participativo, reflexivo, relevante, creativo, equitativo, eficiente y eficaz, promoviendo el liderazgo grupal sin exclusión, donde se integren y complementen cada uno de los agentes educativos.

\section{Referencias}

Aguerrondo, I. (1993). La calidad de la educación: ejes para su definición y evaluación. Revista Interamericana de Desarrollo Educativo, 37 (116), pp. 561-758. Recuperado de: http://www. oei.es/calidad2/aguerrondo.htm

Amadio, M., Opertti, R. \& Tedesco, J. (2014). Un currículo para el siglo XXI: Desafíos, tensiones y cuestiones abiertas. Documentos de Trabajo. Investigación y Prospectiva en Educación, 9. París: UNESCO.

Bambrick-Santoyo, P. (2012). Leverage Leadership: A practical guide to build exceptional schools. San Francisco, California: Jossey-Bass.

Coleman, J., Campbell, E., Hobson, C., McPartland, F., Mood, A., Weinfeld, F. \& York, R. (1996). Equality of educational opportunity. Washington: U.S. Government Printing Office. 
Delors, J., Mufti, I., Amagi, I., Carneiro, R., Chung, F., Geremek, B., Gorham, W., Kornhauser, A., Manley, M., Padrón-Quero, M., Savané, M. A., Singh, K., Stavenhagen, R., Won-Suhr, M. y Nanzhao, Z. (2000). La educación encierra un tesoro. Recuperado de: http://www.unesco. org/education/pdf/DELORS S.PDF

Elmore, R. (2000). Building a new structure forschool leadership. Washington: The Albert Shanker Institute. Elmore, R. (2010). Mejorando las escuelas desde la sala de clases. Santiago, Chile: Fundación Chile.

Estudio Internacional de Progreso en Comprensión Lectora y Estudio Internacional de Tendencias en Matemáticas y Ciencias (PIRLS - TIMSS). (2011). Estudio internacional de progreso en comprensión lectora, matemáticas y ciencias. Volumen I: Informe español. Recuperado de: http://www.mecd.gob.es/dctm/inee/internacional/pirlstimss2011vol1-1. pdf?documentld=0901e72b81825be4

Falconi, O. (2008). Aportes para la gestión del currículo y las políticas educativas. (Conferencia en la ciudad de Paraná). Recuperado de: http://www.entrerios.gov.ar/CGE/2010/primaria/ files/2011/02/Conferencia-de-Octavio-Falconi.pdf

Gairín, J. (2008). Autonomía, calidad y evaluación. En: Secretaria General Técnica (ed). La autonomía de los centros escolares. Madrid: Subdirección General de Información y Publicaciones.

Goe, L. (2007). The link between teacher quality and student outcomes: A research synthesis. Washington: National Comprehensive Center for Teacher Quality.

Goe, L. \& Stickler, L. (2008). Research and policy brief: Teacher quality and student achievement: Making the most of recent research. Washington: National Comprehensive Center for Teacher Quality.

Hallinger, P. \& Murphy, J. (1985). Assessing the instructional leadership behavior of principals. Elementary School Journal, 86(2), 217-248. Recuperado de: http://www.ascd.org/ASCD/ pdf/journals/ed lead/el 198709 hallinger.pdf

Leithwood, K., Day, C., Sammons, P., Harris, A. \& Hopkins, D. (2006). Successful school leadership: What it is and how it influences pupil learning. Nottingham: National College for School Leadership and Department for Education and Skills.

Manzi, J., González, R. \& Zun, Y. (2011). La evaluación docente en Chile. Santiago: Facultad de Ciencias Sociales, MIDE UC.

Marzano, R. (2003). What works in schools. Translating research into action. Alexandria, VA: Association for Supervision and Curriculum Development.

Marzano, R. (2012). Research basis for the Marzano school leadership evaluation model. Florida: Learning Sciences Marzano Center for teacher and leadership evaluation.

Marzano, J., Pickering, D. \& Heflebower, T. (2011). The Highly Engaged Classroom. Bloomington: Marzano Research Laboratory. 
McKinsey \& Company. (2007). How the world's best-performing school systems come out on top. Recuperado de: http://mckinseyonsociety.com/downloads/reports/Education/ Como hicieron los sistemas educativos.pdf

Ministerio de Educación Chile. (2004). Reglamento sobre evaluación docente. Recuperado de: $\quad$ http://www.cpeip.cl/usuarios/cpeip/File/2013\%20documentospsp/ reglamentoevaluaciondocente.pdf

Ministerio de Educación Chile. (2012). Medidas de segregación escolar: discusión para el caso chileno. Santiago: MINEDUC, Centro de Estudios.

Ministerio de Educación Chile. (2013a). Calidad y equidad en el desempeño escolar: simulación de resultados de mejoramiento. Santiago: MINEDUC, Centro de Estudios.

Ministerio de Educación Chile (2013b). Estándares indicativos de desempeño. Santiago: Agencia de la Calidad de la Educación.

Murillo, F.\& Krichesky, G. (2012). El proceso del cambio escolar. Una guía para impulsary sostenera mejora de las escuelas. Revista lberoamericana sobre Calidad, Eficacia y Cambio en Educación, 10 (1), 27-43. Recuperado de: http://www.redalyc.org/articulo.oa?id=55123361003

Murphy, J. (1990). Principal instructional leadership. Advances in Educational Administration: Changing pespectives on the school, 1, 163-200. Greenwitch: JAL.

Observatorio Chileno de Políticas Educativas (OPECH). (2006). Mejoramiento de la Calidad de la Educación: (mucho) más allá de las pruebas estandarizadas. Santiago, Chile: Universidad de Chile.

Oficina Regional de Educación de la UNESCO para América Latina y el Caribe (OREALC/ UNESCO). (2007). El Derecho a una educación de calidad para todos en América Latina y el Caribe. Revista Iberoamericana sobre Calidad, Eficacia y Cambio en Educación, 5 (3), 1-21. Recuperado de: http://www.rinace.net/arts/vol5num3/art1.htm

Organización para la Cooperación y el Desarrollo Económico (OCDE). (2004). Revisión de políticas nacionales en educación en Chile. Recuperado de: http://www.facso.uchile.cl/psicologia/ epe/ documentos/GT cultura escolar politica educativa/recursos\%20bibliograficos/ articulos\%20relacionados/oecd(2004)revisiondepoliticaseducacionenchile.pdf

Organización de Estados Iberoamericanos (OEI). (2008). Metas educativas 2021: la educación que queremos para la generación de los bicentenarios. Madrid: OEI.

Organización para la Cooperación y el Desarrollo Económico (OCDE). (2013). Teaching and learning international survey (TALIS). Recuperado de: http://www.oecd.org/edu/ school/43057468.pdf

Organización de las Naciones Unidas para la Educación, la Ciencia y la Cultura (UNESCO). (2000). La educación encierra un tesoro. Informe de la Comisión Internacional sobre la Educación para el Siglo XXI. París: UNESCO. 
Organización de las Naciones Unidas para la Educación, la Ciencia y la Cultura (UNESCO). (2014). Medium-Term strategy 2014-2021, Laying foundations for equitable lifelong learning for all. Hamburg: UIL.

Rivkin, S., Hanushek, E. \& Kain, J. (2005). Teachers, schools, and academic achievement. Econometrica, 73 (2). Recuperado de: http://www.econ.ucsb.edu/ jon/Econ230C/ HanushekRivkin.pdf

Robinson, V., Hohepa, M. \& Lloyd, C. (2009). School leadership and student outcomes: Identifying what works and why. Nueva Zelandia: Ministry of Education.

Senge, P. (1990). La quinta disciplina. El arte y la práctica de la organización abierta al aprendizaje. Barcelona: Granica.

Valenzuela, J., Bellei, C., \& Ríos, D. (2011). Segregación escolar en Chile. En: Martinic, S. y Elacqua, G. (ed.) Fin de ciclo: Cambios en la gobernanza del sistema educativo. Facultad de Educación, Pontificia Universidad Católica de Chile-OREAL/UNESCO. Recuperado de: http://unesdoc. unesco.org/images/0019/001905/190544S.pdf

Weber, J. (1996). Leading the instructional program. En:Smith, S.y Piele, P. (eds.). Schoolleadership. Oregon: Clearinghouse of Educational Management. Recuperado de: http://eric. ed.gov/?id=ED309513

Weinstein, J. (2009). Liderazgo directivo, asignatura pendiente de la Reforma Educacional Chilena. Revista Estudios Sociales, 117, 123-148. Recuperado de: http://www.ceppe.cl/ images/stories/recursos/publicaciones/Jose\%20Weinstein/Weinstein LIDERAZGO DIRECTIVO doc.pdf

Weinstein, J. \& Muñoz, G. (2012). ¿Qué sabemos sobre los directores de escuela en Chile? Santiago, Chile: Centro de Innovación en Educación de Fundación Chile y Centro de Estudios y Políticas y Prácticas en Educación (CEPPE). 